\title{
A Comparison of Needle Bending Models
}

\author{
Ehsan Dehghan, Orcun Goksel, and Septimiu E. Salcudean \\ Department of Electrical and Computer Engineering, \\ University of British Columbia, Vancouver, Canada \\ \{ehsand, orcung, tims\}@ece.ubc.ca
}

\begin{abstract}
Modeling the deflection of flexible needles is an essential part of needle insertion simulation and path planning. In this paper, three models are compared in terms of accuracy in simulating the bending of a prostate brachytherapy needle. The first two utilize the finite element method, one using geometric non-linearity and triangular plane elements, the other using non-linear beam elements. The third model uses angular springs to model cantilever deflection. The simulations are compared with the experimental bent needle configurations. The models are assessed in terms of geometric conformity using independently identified and pre-identified model parameters. The results show that the angular spring model, which is also the simplest, simulates the needle more accurately than the others.
\end{abstract}

\section{Introduction}

The insertion of needles into soft tissue is an essential part of many medical interventions such as biopsy and brachytherapy. Due to tissue deformation and needle bending during insertion, target displacement, and insufficient feedback from medical imaging, reaching a target location with the needle requires significant skill, training, and experience of the performing physician. Therefore, physical simulators and path planning systems for needle insertion are areas of need for accurate models of flexible needles.

Due to the complexity of interactions between needles and tissue, needle insertion simulation cannot be accommodated using a combined mesh. Therefore, two separate models for the tissue and the needle are usually employed [1,2,3,4 .

Tissue deformation and target displacement during rigid needle insertion have been studied in 2D [1,2]. Prior work on flexible needle simulation generally assumed needle bending in 2D. DiMaio and Salcudean [2] simulated the needle as an elastic material using the finite element method (FEM) with geometric nonlinearity. This method was extended to 3D using 4-node tetrahedral elements by Goksel et al. 4].

Webster et al. 3] identified a non-holonomic model for a highly flexible needle to simulate the effect of bevel tip on needle motion in tissue. The assumption of high flexibility is unfortunately not valid for many conventional needles such as the ones used for epidural blocks and prostate brachytherapy. This model was later used in [5, 6] for path planning of a needle in 2D tissue. Glozman 
and Shoham [7] used linear beam elements to simulate needle bending for needle steering. This approach is relatively simple and fast but the elements are not rotation invariant and do not preserve needle length during large deformations. Friction and cutting forces on the needle during insertion have also been studied [8,9].

Because they are easy to simulate, angular springs have been widely used in cloth and hair modeling [10 and 3D modeling of vibration and deformation [1] . Most of these contributions used complex dynamic models in 3D with various approximations to angular spring behavior (i.e. linear or quadratic). This paper focuses on the physical validity and the modeling accuracy of this method for static deformations of thin rods, and specifically for the deformation of a brachytherapy needle. Different models are assessed in terms of modeling accuracy. The model simulation results are compared with the experimental data. Although the models in this paper are described and validated only in 2D and with a single tip force, they can be easily extended to $3 \mathrm{D}$ with multiple forces along the needle. The following section introduces the three different models studied.

\section{Methods}

\subsection{Finite Element Method Using Triangular Elements}

The finite element method with triangular elements can be used to predict needle bending. If the needle elastic material is assumed to be linear (mechanical linearity), the linear geometry assumption leads to a set of linear algebraic equations between nodal forces and displacements. While linearity can be exploited for real time implementation, the linear geometry assumption is not valid for needle deformation, due to large rotations and displacements [2].

Using the non-linear geometric relation between strain and displacement leads to a set of non-linear algebraic equations

$$
\underline{f}=\hat{\mathbf{K}}(\underline{u}) \underline{u}
$$

where $\underline{u}$ and $\underline{f}$ are the vectors of nodal displacements and forces, respectively. This non-linear formula can compensate for axial displacements and preserves the needle length during large lateral deformations. Although computationally expensive compared to the solution of the linear model, the Newton-Raphson method [12] can be employed to solve the set of non-linear algebraic equations in (11). Simulations show that this method is stable and converges in a few iterations. However, offline computations and condensation cannot be used.

\subsection{Finite Element Method Using Non-linear Beam Elements}

Another choice of element suitable for needle modeling is the Euler-Bernoulli beam element, which employs linear and cubic interpolation functions for the axial displacement $u$ and the transverse displacement $w$, respectively. The nodal 
variables at node $p$ are the longitudinal displacement $u^{p}$, the transverse deflection $s_{1}^{p}$ and its derivative $s_{2}^{p}$, such that $s_{1}^{p}=w$ and $s_{2}^{p}=-d w / d x$. Considering nonlinear geometry, the equilibrium equation can be written as [12]:

$$
\left[\begin{array}{ll}
\mathbf{K}_{(2 \times 2)}^{11} & \mathbf{K}_{(2 \times 4)}^{12} \\
\mathbf{K}_{(4 \times 2)}^{21} & \mathbf{K}_{(4 \times 4)}^{22}
\end{array}\right]\left[\begin{array}{l}
\underline{u} \\
\underline{s}
\end{array}\right]=\left[\begin{array}{l}
\underline{f}_{1} \\
\underline{f}_{2}
\end{array}\right]
$$

where $\underline{f}_{1}$ is the vector of axial forces and $\underline{f}_{2}$ is the vector of lateral forces and torques on the nodes. $\mathbf{K}^{i j}$ is a function of the material and geometric properties of the beam and the nodal displacements and rotations. This non-linear set of equations can be easily solved using the Picard iterative method 12. Reduced integration should be used in integrating the stiffness matrix in (2) to avoid membrane locking in the element due to large deformations [12].

The linear geometry assumption yields the linear beam element formulation, which separates displacements in axial and transverse directions. This model approximates the transverse deflections with a simple third order curve. Although the linear beam element stiffness matrix is constant and makes offline computation possible, it cannot preserve the needle length during deformation.

\subsection{Angular Spring Model}

This method models the cantilever using a number of rigid rods connected via rotational springs (see Fig.1(right)). Consider a short section of length $L$ of a bent cantilever under a constant bending moment $M$ (all in 2D). Fig.1(left) shows the neutral axis of the bent cantilever and its bending radius $\rho$. Let $c$ be the distance of the neutral axis from the outer fibre. From similar triangles we obtain the following:

$$
\frac{L+\delta}{L}=\frac{\rho+c}{\rho} \Rightarrow \frac{\delta}{L}=\frac{c}{\rho}
$$

Substituting (3) in the definition of outer fibre strain and using the fact that $L=\theta \rho$ leads to:

$$
\frac{\delta}{L}=\epsilon=\frac{M c}{E I} \Rightarrow \frac{1}{\rho}=\frac{M}{E I}=\frac{\theta}{L}
$$

where $E$ is the stiffness and $I$ is the moment of inertia. With respect to the initial unbent orientation, the angle $\alpha$ of deflection caused by the bent section being

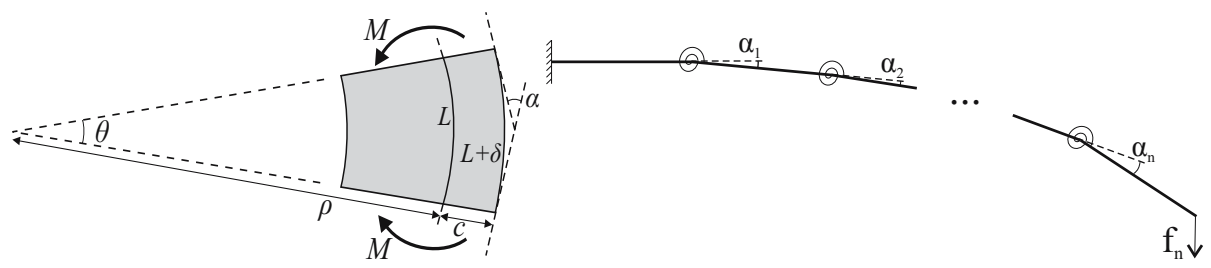

Fig. 1. A short section of a bent cantilever (left), and (right) The angular spring model for a needle 
analyzed is equal to $\theta$. Thus, using (4) the linear relation between the bending moment and the deflection angle is written as $M=k \alpha$ where $k=2 E I / L$. While the definition of $k$ obtained for an infinitesimal length may not hold for the discretized model described next, continuum analysis leads to an expected linear relation between $M$ and $\alpha$.

The angular spring model is composed of line segments connected via springs. In a model with $n+1$ segments the equation between forces and joint angles is:

$$
\left[J_{1}^{T}\left(\alpha_{1}\right)\left|J_{2}^{T}\left(\alpha_{1}, \alpha_{2}\right)\right| \cdots \mid J_{n}^{T}\left(\alpha_{1}, \cdots, \alpha_{n}\right)\right]\left[\begin{array}{c}
f_{1} \\
\vdots \\
f_{n}
\end{array}\right]=\mathbf{K}\left[\begin{array}{c}
\alpha_{1} \\
\vdots \\
\alpha_{n}
\end{array}\right]
$$

where $\alpha_{i}$ are the joint angles, $J_{i}^{T}$ is the transposed conventional serial link Jacobian matrix up to link $i, f_{i}$ is the nodal force on the node $(i+1)$ and $\mathbf{K}$ is a diagonal matrix of spring constants. Since the material is the same throughout the shaft, all springs are assigned the same constant. Despite their non-linearity, these equations can be solved iteratively by fast and simple forward vectoralgebraic equations. If the needle is in a posture with values $\left(\alpha_{1}, \cdots, \alpha_{n}\right)$ at the $t^{\text {th }}$ iteration, the next values for the angles are:

$$
\alpha_{i}^{t+1}=\sum_{j \geq i}^{n} M_{i j}^{t} / k
$$

where $M_{i j}^{t}$ is the torque applied by force $f_{j}$ on joint $i$ in $t^{\text {th }}$ iteration. The procedure converges fast, typically in 2 to 5 iterations.

\section{Experiment and Simulations}

In order to compare the models with the actual needle bending, an 18 gauge $20 \mathrm{~cm}$ brachytherapy needle (Bard, NJ, USA), consisting of a steel stylet sliding inside a steel cannula, was used in an experiment. For simplicity the needle is modeled as a simple bar with a single unknown parameter that is identified by fitting the model to experimental data acquired by bending the needle with a force at the tip. The Young's modulus for the finite element model and the spring constant for the angular spring model are the unknown model parameters to be identified. Since this simplified model represents the combination of the cannula and stylet, the identified Young's moduli are not equal to the Young's modulus of steel and the identified spring constants do not satisfy the definition of $k$ in Section 2.3.

To identify the model parameters, two cost functions of the lateral tip position error and the area error between the models and the real needle were minimized independently. The former is the vertical difference between the simulated and the experimental tip position. The latter is the integral area lying between the simulated and the experimental needle shafts. The identified parameters were used in the model to simulate the bending of the needle. 

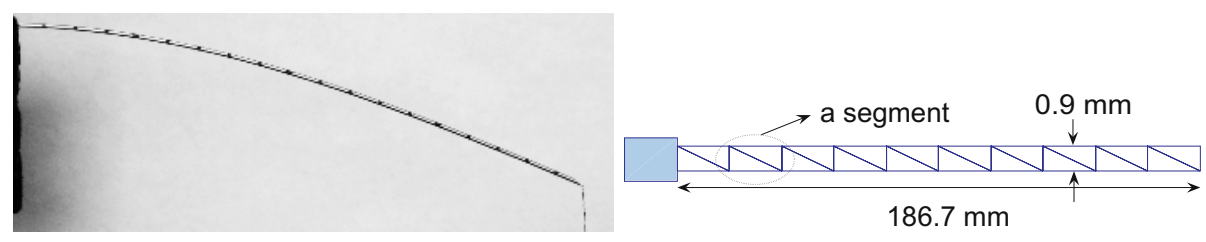

Fig. 2. Left: Needle shaft with the scales hanging at the tip. Right: Discretized needle using a $11 \times 2$ nodes mesh.

Table 1. Tip deflection for various lateral tip forces

\begin{tabular}{|l|r|r|r|r|r|r|r|}
\hline Force $[\mathbf{m N}]$ & 63 & 113 & 163 & 213 & 263 & 313 & 413 \\
\hline Deflection [mm] & 8.3 & 14.5 & 20.9 & 27.0 & 33.0 & 39.0 & 50.2 \\
\hline
\end{tabular}

The brachytherapy needle was horizontally clamped at its base while a vertical force was applied on its tip. The clamp decreased the effective needle length to $18.7 \mathrm{~cm}$. Different vertical tip forces were achieved by hanging different scaling weights of $5,10,15,20,25,30$, and $40 \mathrm{~g}$ on a tiny hook at the tip (see Fig.(2). The stage setup used for hanging weighted $1.3 \mathrm{~g}$. The vertical needle tip force was varied between $63 \mathrm{mN}$ and $413 \mathrm{mN}$. Images of the needle were taken on a white background with a digital camera, the shutter of which was controlled by computer. The tip deflection from nominal axis is given in Table1 for each applied force.

For FEM with triangular elements, meshes of $11 \times 2$ and $21 \times 2$ nodes were used to discretize the needle as shown in Fig.22. Plain stress analysis was performed with a thickness of $0.9 \mathrm{~mm}$, and taking the Poisson's ratio as 0.3 , equal to the Poisson's ratio of steel. The Young's modulus was identified to fit this model to the experimental data. Similarly, 10 and 20 segment models were used for both the non-linear beam element and the angular spring models. The differences between identified parameters from two different optimization cost functions were less than $2 \%$. Therefore, throughout the rest of this paper we are referring to the values that were identified using the lateral tip-error minimization.

In the triangular FEM and the beam models, the mean values of the identified Young's moduli for different forces were used for simulation and verification. For the triangular element model, this mean value increased with the number of segments. Since the strain is assumed to be constant inside this element a lower Young's modulus (softer material) is needed to yield the same amount of deformation when fewer elements are used. The identified Young's moduli for the non-linear beam element model were constant for any number of elements.

Using the angular spring model, the mean value of the spring constants increased with the change in the number of segments from 10 to 20 and 50 as expected. Normalizing these values with segment length did not show a constant number. In this model the spring constants were identified directly and then used in the simulation phase. 


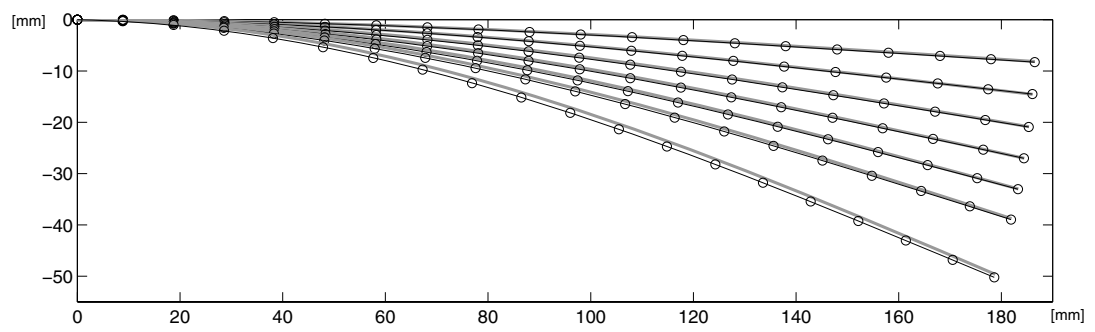

(a) Triangular element with $21 \times 2$ nodes.

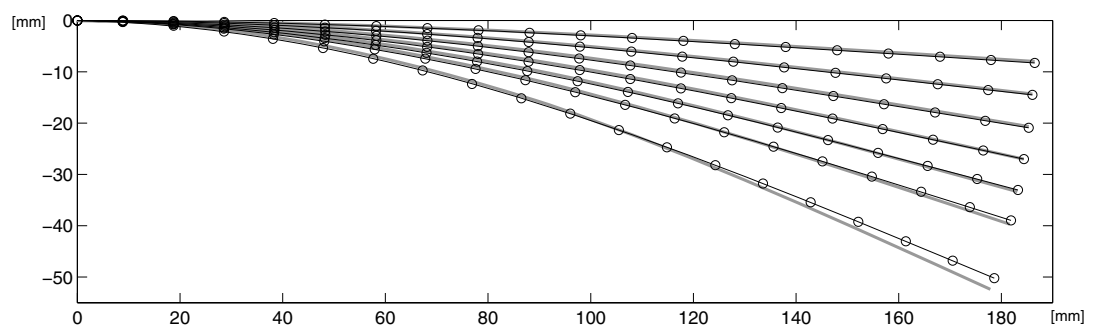

(b) The non-linear beam element with 20 segments.

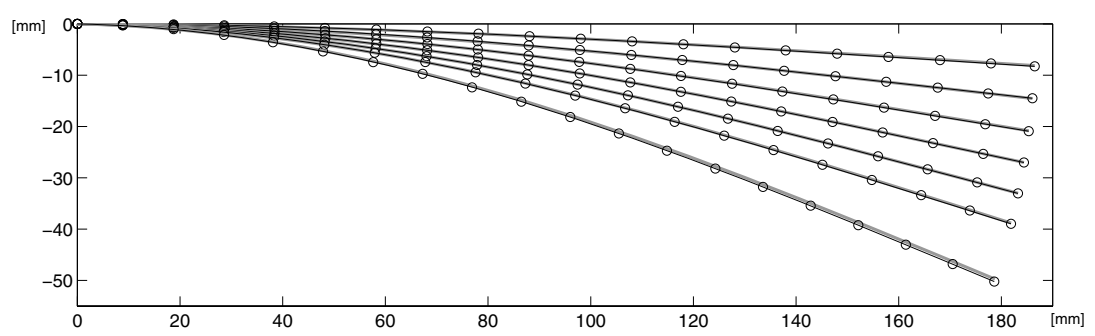

(c) Angular spring model with 20 segments.

Fig. 3. The experimental data (circles connected with dashed lines) and the simulated needle (solid lines) using the mean value of identified parameters

In the next step, the needle bending was simulated for a range of tip loads using the mean value of the identified parameters. The identified parameters for all the models did not deviate from their mean values by more than $4 \%$. Figure 3 shows the simulated and the real needle configuration for the three models. For each simulation a tip and an area error are reported in Fig.4.

\section{Discussion}

Comparing the two FEM based models, the triangular element model has a slightly higher accuracy than the non-linear beam model. However, the tip position error is less than the needle diameter in most cases. Although the elements of both models have 6 degrees of freedom, modeling a rod using triangular elements requires more elements than in the beam element approach. This leads 

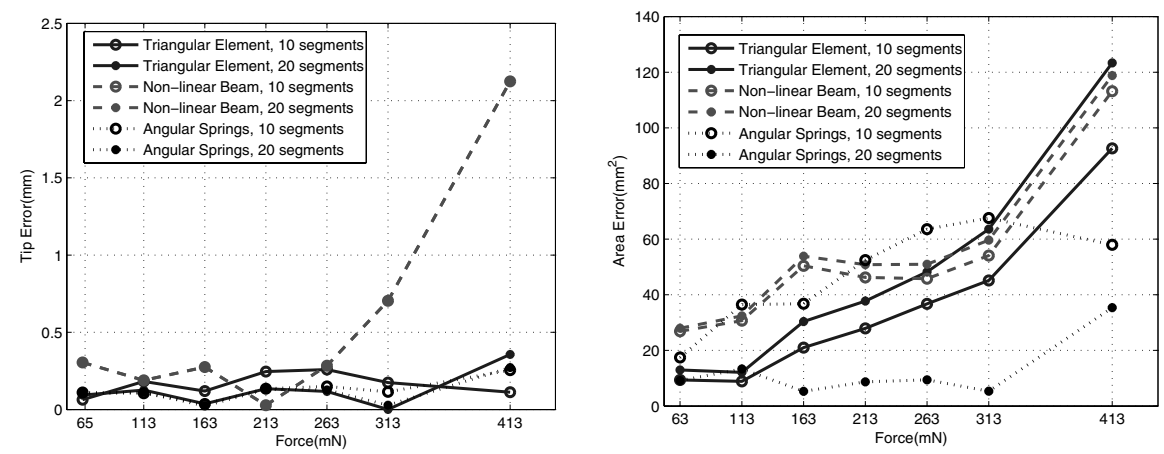

Fig. 4. Comparison of (Left) lateral tip position and (Right) area errors of different models with 10 and 20 segments using the mean value of parameters

to larger matrices. For example modeling a needle using a mesh of $n \times 2$ nodes using triangular elements leads to a $4 n \times 4 n$ tangent stiffness matrix while using $n$ nodes as beam elements leads to a $3 n \times 3 n$ one. In addition, the fact that the beam model results are not changing with the number of nodes can be utilized for adaptive meshing in needle insertion simulation to achieve higher speed without loss of accuracy. In adaptive meshing an element is added as the needle penetrates the tissue and makes contact with more tissue mesh nodes [7].

Extension of the non-linear beam model to 3D is straightforward. Assuming that the axial moments and bending are negligible, each element in 3D has 5 degrees of freedom leading to a $10 \times 10$ tangent stiffness matrix that can be computed as in the $2 \mathrm{D}$ case.

Extension of the triangular element model to 3D leads to tetrahedral elements with $12 \times 12$ tangent stiffness matrices. Meshes used in this paper for the triangular element models are asymmetric. For more accurate simulations, symmetric meshes should be used which require more elements and proper design [4]. In contrast to this, the non-linear beam and angular spring models are always symmetric. Therefore, using triangular or tetrahedral symmetric models in $2 \mathrm{D}$ or $3 \mathrm{D}$ requires a significantly higher number of elements and is computationally more demanding than modeling the needle with non-linear beam elements or with angular spring models.

Of the three models considered, the angular spring model gives the most accurate results, as shown in Fig.44. It is also computationally more efficient. Extension to 3D is straightforward as presented for hair models in [10] .

While not considered explicitly in this paper, the effect of tissue forces that are distributed along the needle, not only at the tip, can be modeled in a similar manner, for both the planar and 3D cases.

\section{Conclusion}

Three different models for simulating the flexibility of a brachytherapy needle have been demonstrated in $2 \mathrm{D}$. An experimental setup was designed to verify the 
models. Model parameters (Young's moduli and spring constants) were identified using the collected data and their mean values were used to simulate needle bending arising from vertical needle tip forces. The fit of the simulations to the experiments was reported in terms of tip error and mismatching area error.

Based on the results one can conclude that the angular spring model is the easiest to implement, the fastest to compute and the most accurate in simulating needle flexibility in $2 \mathrm{D}$.

In the future, the needle bending models will be validated in $3 \mathrm{D}$ using the ultrasound images exploiting the methods introduced in [13] .

\section{References}

1. Alterovitz, R., Goldberg, K., Pouliot, J., Taschereau, R., Hsu, I.C.: Needle insertion and radioactive seed implantation in human tissue: Simulation and sensitivity analysis. In: Proc. IEEE Int. Conf. Rob. Autom. Volume 2. (2003) 1793-1799

2. DiMaio, S., Salcudean, S.: Interactive simulation of needle insertion models. IEEE Trans. Biomed. Eng. 52 (2005) 1167-1179

3. Webster III, R., Cowan, N., Chirikjian, G., Okamura, A.: Nonholonomic modeling of needle steering. In: Proc. Int. Symp. on Exp. Robotics. (2004) 3337-3343

4. Goksel, O., DiMaio, S.P., Salcudean, S.E., Rohling, R., Morris, J.: 3D needle-tissue interaction simulation for prostate brachytherapy. In: Proc. MICCAI. (2005)

5. Alterovitz, R., Goldberg, K., Chirikijan, G., Okamura, A.: Steering flexible needle under markov motion uncertainity. In: IEEE Int. Conf. Intel. Rob. Sys. (2005) $120-125$

6. Alterovitz, R., Goldberg, K., Okamura, A.: Planning for steerable bevel-tip needle insertion through 2D soft tissue with obstacles. In: Proc. IEEE. Int. Conf. Rob. Autom. (2005) 1652-1657

7. Glozman, D., Shoham, M.: Flexible needle steering and optimal trajectory planning for percutaneous therapies. In: Proc. MICCAI. (2004) 137-144

8. DiMaio, S.P., Salcudean, S.E.: Needle insertion modeling and simulation. IEEE Trans. Robotics and Automation 19 (2003) $864-875$

9. Simone, C., Okamura, A.: Modeling of needle insertion forces for robot-assisted percutaneous therapy. In: Proc. IEEE Int. Conf. Rob. Autom. (2002) 2085-2091

10. Anjyo, K., Usami, Y., Kurihara, T.: Simple method for extracting the natural beauty of hair. Computer Graphics (ACM) 26 (1992) 111-120

11. Anshelevich, E., Owens, S., Lamiraux, F., Kavraki, L.E.: Deformable volumes in path planning applications. In: Proc. IEEE Int. Conf. Rob. Autom. (2000) $2290-2295$

12. Reddy, J.N.: An Introduction to Nonlinear Finite Element Analysis. Oxford University Press (2004)

13. Ebrahimi, R., Okazawa, S., Rohling, R., Salcudean, S.: Hand-held steerable needle device. In: Proc. MICCAI. (2003) 223-230 